\title{
Multifield Dirac-Born-Infeld inflation and non-Gaussianities
}

\author{
Min-xin Huang \\ Department of Physics, CERN-Theory Division, CH-1211 Geneva 23, Switzerland
}

Gary Shiu and Bret Underwood

Department of Physics, University of Wisconsin, Madison, Wisconsin 53706, USA

(Received 8 October 2007; published 15 January 2008)

\begin{abstract}
We analyze the trajectories for multifield Dirac-Born-Infeld inflation, which can arise in brane inflation models, and show that the trajectories are the same as in typical slow roll inflation. We calculate the power spectrum and find that the higher derivative terms of the Dirac-Born-Infeld action lead to a suppression of the contribution from the isocurvature perturbations. We also calculate the bispectrum generated by the isocurvature perturbation, and find that it leads to distinctive features.
\end{abstract}

DOI: 10.1103/PhysRevD.77.023511

PACS numbers: 98.80.Cq, 11.25.Mj

\section{INTRODUCTION}

Scalar field theories with noncanonical kinetic terms provide novel realizations of the inflationary paradigm [1]. One interesting class of such models which have been studied extensively in recent years is DBI inflation [2,3], characterized by kinetic terms which arise from the Dirac-Born-Infeld (DBI) action. A particularly appealing phenomenological feature of DBI inflation is that it can lead to strong and unique non-Gaussian signatures in the cosmic microwave background (CMB) [3,4]. The DBI action is ubiquitous in string theory, e.g., as an effective theory for world volume degrees of freedom on branes. When embedded in brane inflation [5], the inflaton field in DBI models can be given a natural geometrical interpretation as the position of a $D$-brane in extra dimensions. DBI inflation arises when the $D$-brane moves in a highly warped region of the internal space where the speed limit is small, and reduces to the usual slow-roll brane inflation (with canonical kinetic term) when the brane is moving nonrelativisitically with respect to the local warp factor. Since the position of the brane in each compact direction is described by a scalar field, brane inflation is naturally a multifield inflationary model.

Multifield models are characterized by their trajectories in field space and can in general be decomposed into an adiabatic field, which parametrizes motion along the trajectory, and isocurvature fields, which describe the directions perpendicular to the trajectory. Features in the trajectory, such as a sharp turn, can convert isocurvature perturbations into adiabatic/curvature perturbations (even on superhorizon scales) and can give rise to interesting features in the primordial power spectrum and nonGaussianity.

In this paper we study the effects of multiple fields in DBI inflation. In particular, we study the multifield DBI trajectories and show that they are identical to the usual slow-roll multifield case in which the trajectory is dominated by the field with the largest slope of the potential. We calculate the power spectrum for multiple DBI fields in the limit the trajectory makes a sharp turn and show that the contribution of the isocurvature perturbations to the power spectrum is suppressed by the sound speed. Finally, we calculate the non-Gaussianity in the sharp turn limit and find that the non-Gaussianity is dominated at leading order in the sound speed by the usual single field DBI contribution, but has new multifield features at subleading order. We conclude by commenting on the impact of our results for DBI model building.

\section{MULTIFIELD EQUATIONS OF MOTION}

Consider a 10-dimensional warped throat background with the metric

$$
d s^{2}=\tilde{f}^{-1 / 2}(y) g_{\mu \nu} d x^{\mu} d x^{\nu}+\tilde{f}^{1 / 2}(y) \tilde{g}_{m n} d y^{m} d y^{n}
$$

common to type IIB string compactifications [6,7], where $\tilde{f}(y)$ is called the warp factor of the throat which can in principle depend on all of the coordinates of the internal space $\vec{y}$.

A D3-brane in this background is described by (to lowest order in string coupling and to all orders in $\alpha^{\prime}$ ),

$$
\begin{aligned}
S_{\mathrm{DBI}}= & -\int d^{4} x \sqrt{-g} \\
& \times\left[\frac{1}{f\left(\phi_{i}\right)}\left(\sqrt{1+f\left(\phi_{i}\right) g^{\mu \nu} \sum_{i} \partial_{\mu} \phi_{i} \partial_{\nu} \phi_{i}}-1\right)\right. \\
& \left.+V\left(\phi_{i}\right)\right]
\end{aligned}
$$

where the warp factor is rescaled $f\left(\phi_{i}\right)=T_{D 3}^{-1} \tilde{f}\left(y\left(\phi_{i}\right)\right)$, and the real canonical scalar fields associated with the motion of the brane are given by

$$
\phi_{i} \equiv T_{D 3}^{1 / 2} y_{i}
$$

where $i=1, \ldots 6$. The potential $V\left(\phi_{i}\right)$ can arise, for example, from interactions with $\bar{D} 3$-branes, $D 7$-branes, or from the breaking of the local isometries of the compact 
space, and we will leave it to be unspecified for the moment.

We will define the sound speed during inflation to be the inverse of the "Lorentz factor" of the DBI action for spatially homogeneous fields,

$$
c_{s}=\sqrt{1-f\left(\phi_{i}\right) \sum_{i} \dot{\phi}_{i}^{2}}
$$

We will be interested in the small sound speed limit $c_{s} \ll 1$ where the non-Gaussianity is observable. In the following we will use the convention that the $y_{i}$ measure the distance from IR "tip" of the throat and that the D3 brane moves towards the tip, so $\dot{\phi}_{i}<0$.

Consider a FRW universe with four-dimensional metric,

$$
d s^{2}=-d t^{2}+a(t)^{2} \sum_{i=1}^{3} d x_{i}^{2}
$$

The Friedman equation and equations of motion for the fields $\phi_{i}$ are (with $H=\frac{\dot{a}}{a}$ )

$$
\begin{gathered}
3 M_{p}^{2} H^{2}=\frac{1}{f\left(\phi_{i}\right)}\left(\frac{1}{c_{s}}-1\right)+V\left(\phi_{i}\right) \\
\frac{1}{a(t)^{3}} \frac{d}{d t}\left(a(t)^{3} \frac{\dot{\phi}_{i}}{c_{s}}\right)=-\frac{\partial}{\partial \phi_{i}}\left(V\left(\phi_{i}\right)+f\left(\phi_{i}\right)^{-1}\left(c_{s}-1\right)\right)
\end{gathered}
$$

Distributing the time derivative, the equation of motion (7) can also be written

$$
\ddot{\phi}_{i}+3 H \dot{\phi}_{i}-\frac{\dot{c}_{s}}{c_{s}} \dot{\phi}_{i}+c_{s} \partial_{\phi_{i}}\left(V+\frac{\left(c_{s}-1\right)}{f}\right)=0 .
$$

Clearly the equation of motion for a homogeneous scalar field with a canonical kinetic term is obtained from (7) and (8) in the limit $c_{s} \rightarrow 1$.

We define the multifield DBI inflationary parameters as [4]

$$
\begin{gathered}
\epsilon \equiv-\frac{\dot{H}}{H^{2}} \\
\eta_{i j} \equiv M_{p}^{2} c_{s} \frac{\partial_{\phi_{i}} \partial_{\phi_{j}} V}{V} .
\end{gathered}
$$

When these parameters are much smaller than 1 , the equations of motion (8) take a form similar to that of a slowly rolling field

$$
\begin{aligned}
& 3 H \dot{\phi}_{i}\left(1-\frac{\sum_{j} \tan \theta_{i j} \eta_{i j}+\epsilon}{3}\right)+\partial_{\phi_{i}}\left(V\left(\phi_{i}\right)+f^{-1}\left(c_{s}-1\right)\right) \\
& \approx 3 H \dot{\phi}_{i}+c_{s} \partial_{\phi_{i}}\left(V\left(\phi_{i}\right)+f^{-1}\left(c_{s}-1\right)\right)=0
\end{aligned}
$$

where $\tan \theta_{i j} \approx \partial_{\phi_{j}} V / \partial_{\phi_{i}} V$ to leading order in the DBI inflationary parameters (9) and (10).
As a specific example, for the "standard case" of an AdS warp factor that depends only on one of the fields $f\left(\phi_{1}\right)=\lambda / \phi^{4}$ and a separable potential of mass terms $V=\frac{1}{2} \sum m_{i}^{2} \phi_{i}^{2}$, it can be shown that for small sound speed $c_{s} \ll 1$ and a large mass hierarchy $\left(m_{i} \gg m_{j}\right.$ for some $\left.i, j\right)$ the multifield DBI inflationary parameters are of order $\mathcal{O}(\epsilon)$.

\section{TRAJECTORIES IN MULTIFIELD BRANE INFLATION}

To simplify our analysis we will restrict ourselves to a two field model $\left(\phi_{1}, \phi_{2}\right)$, but it is straightforward to generalize our analysis to any number of fields.

We will parametrize the classical trajectory by an "adiabatic field" $\sigma$ that represents the component of the field motion along the trajectory [8] (see Fig. 1),

$$
\dot{\sigma}=(\cos \theta) \dot{\phi}_{1}+(\sin \theta) \dot{\phi}_{2}
$$

The angle $\theta$ parametrizes the angle the classical trajectory makes with one of the field directions (here chosen to be $\phi_{1}$ ) and should not be confused with the angular position of the $D 3$ brane in the compact space, which is parametrized by the fields $\phi_{i}$. The "entropy field" $s$ transverse to the classical trajectory gives rise to isocurvature fluctuations which are given by

$$
\delta s=-(\sin \theta) \delta \phi_{1}+(\cos \theta) \delta \phi_{2} .
$$

By definition, the entropy field is constant, $\dot{s}=0$.

Using this parametrization, we can rewrite the exact equations of motion (8) as (in particular, the multifield DBI parameters are not necessarily assumed small for

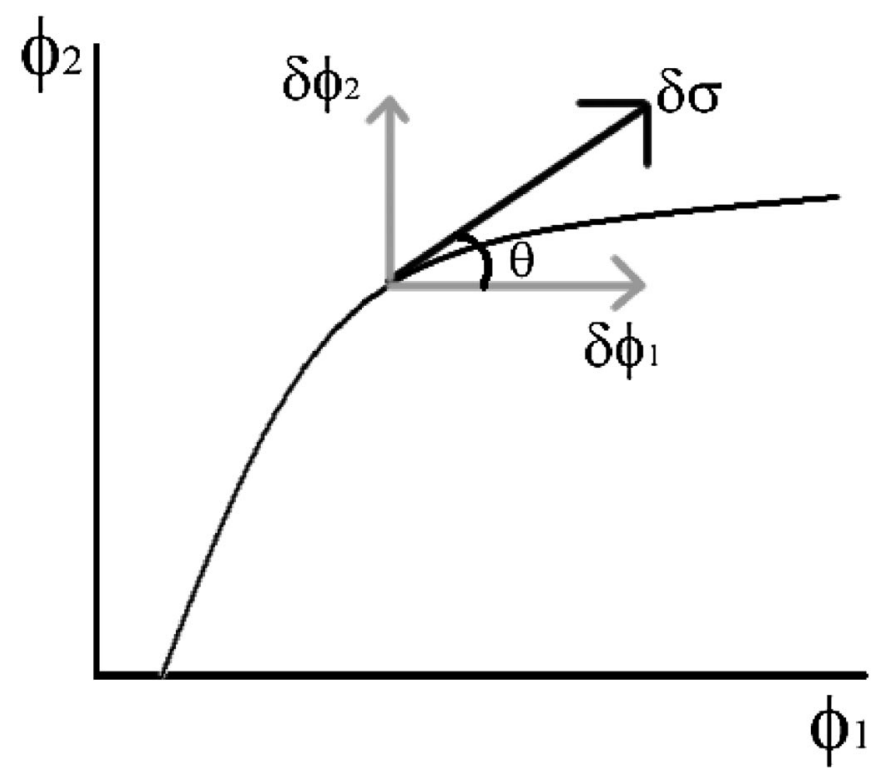

FIG. 1. The trajectory of a multiple field inflationary system can be decomposed into an "adiabatic" field $\sigma$ with components along the trajectory and an "entropy" field $s$ orthogonal to the trajectory. 
this expression),

$$
\begin{gathered}
\frac{\ddot{\sigma}}{c_{s}}+\frac{3 H \dot{\sigma}}{c_{s}}-\frac{\dot{c}_{s}}{c_{s}^{2}} \dot{\sigma}+\partial_{\sigma}\left(V+f^{-1}\left(c_{s}-1\right)\right)=0 \\
\dot{\theta} \dot{\sigma}=\partial_{\phi_{1}}\left(V+f^{-1}\left(c_{s}-1\right)\right) \sin \theta \\
-\partial_{\phi_{2}}\left(V+f^{-1}\left(c_{s}-1\right)\right) \cos \theta .
\end{gathered}
$$

It is clear from (15) that the angle of the trajectory has a "fixed point" trajectory in field space $\dot{\theta} \approx 0$ defined by

$$
\tan \theta_{*}=\frac{\partial_{\phi_{2}}\left(V+f^{-1}\left(c_{s}-1\right)\right)}{\partial_{\phi_{1}}\left(V+f^{-1}\left(c_{s}-1\right)\right)} .
$$

Furthermore this fixed point is stable to leading order when the slope of the potential is positive and dominates over the slope of the warp factor since small variations $\delta \theta$ are driven to zero (notice that $\dot{\sigma}<0$ ). This fixed point can also be seen from the equations of motion (11) in the "DBI slow-roll regime" [e.g. when the multifield DBI parameters (9) and (10) are small],

$$
\tan \theta=\frac{\dot{\phi}_{2}}{\dot{\phi}_{1}} \approx \frac{\partial_{\phi_{2}}\left(V+f^{-1}\left(c_{s}-1\right)\right)}{\partial_{\phi_{1}}\left(V+f^{-1}\left(c_{s}-1\right)\right)} .
$$

We see then that being in the DBI slow-roll regime is equivalent to being at the stable "fixed point" of the trajectory, thus DBI slow-roll is an attractor solution.

Let us examine (16) in more detail. First, we note that for an inflationary solution we require that the potential energy dominates over the kinetic energy, e.g. $V\left(\phi_{i}\right) \gg 1 /\left(c_{s} f\right)$, so that for small $c_{s}, V\left(\phi_{i}\right) \gg c_{s} / f$ is automatically true. The trajectories (16) are now the same as in standard multifield inflation, in particular, the angle of the trajectory

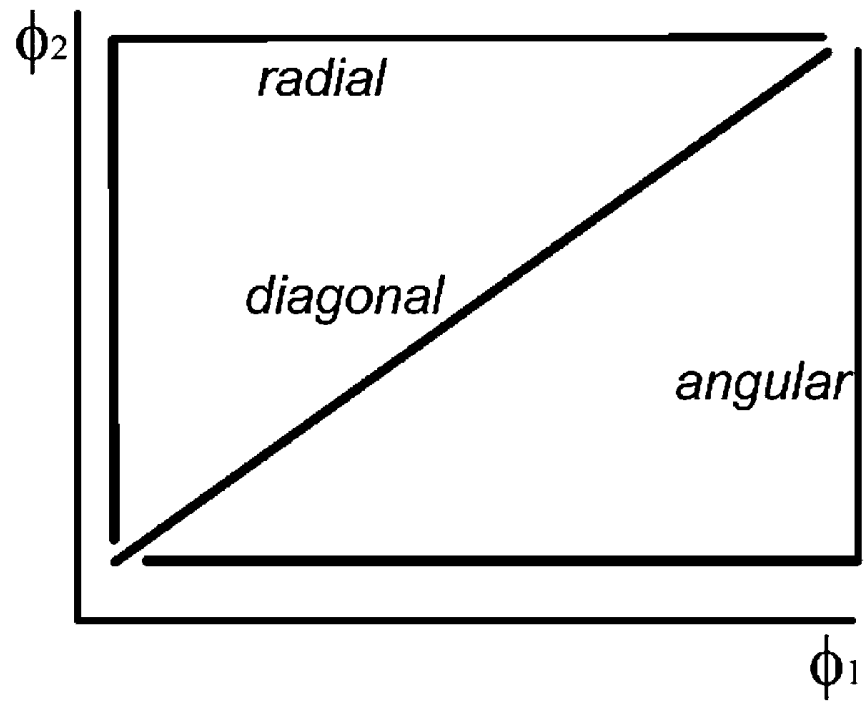

FIG. 2. Multifield models have a number of different trajectories, depending on the curvature of the potential for the fields. The sharpness of the turns is controlled by the ratio of the curvatures. is controlled by the ratio of the curvatures of the potential: the trajectory follows the direction with the largest curvature. When the field with the largest curvature reaches its minimum the trajectory makes a sharp turn in field space, with the sharpness of the turn given by the ratio of the curvatures.

If we express the warped geometry as a cone over an angular base space $X^{5}$,

$$
\tilde{g}_{m n} d y^{m} d y^{n}=d r^{2}+d s_{X^{5}}^{2}
$$

we can identify one of the fields $\phi_{1}$ as the radial coordinate and the other field $\phi_{2}$ as one of the angular coordinates on the base $X^{5}$ of the $D$-brane. In this case we can roughly classify the trajectories based on which field dominates at early times, as shown in Fig. 2: a radially dominated trajectory is when the slope of the potential in the angular direction $\phi_{2}$ is much smaller than the radial direction $\phi_{1}$ and so the trajectory is dominated at early times by motion in the radial direction; a diagonal trajectory is when the slopes of potentials of the two fields are approximately the same and so the trajectory is approximately a diagonal line composed of a linear combination of the radial and angular directions; and a angularly dominated trajectory is when the slope of the potential in the radial direction $\phi_{1}$ is the smallest so the trajectory is dominated at early times by motion in the angular $\phi_{2}$ direction. Clearly the diagonal trajectory does not have a significant turn in field space, and so will not contribute to a generation of curvature perturbations as discussed above. In fact, the diagonaltype trajectory is just a linear combination of the fields and hence can be completely described by a single field, and so we will not consider this possibility.

\section{THE POWER SPECTRUM OF MULTIFIELD DBI INFLATION}

The equation of motion for the curvature perturbation $\mathcal{R} \approx \zeta$ in a multifield model with fields $\phi_{I}$ can be solved exactly using the $\delta N$ formalism [9,10], which states that the curvature perturbation is equal to the difference between the number of e-folds of the classical trajectory $N_{e}$ and the perturbation to the classical trajectory $\mathcal{N}_{e}$,

$$
\zeta=d N_{e}=\mathcal{N}_{e}-N_{e}=\sum_{i} N_{, i}\left(\delta \phi_{i}\right)^{*}
$$

where $N_{, i} \equiv \frac{\partial N_{e}}{\partial \phi_{i}^{*}}$ is the derivative of the number of efoldings with respect to the field evaluated when the mode exits the horizon $\phi_{i}^{*}$. For two fields, the power spectrum is then given by

$$
\begin{aligned}
4 \pi^{2} P_{\zeta}= & \langle\zeta \zeta\rangle=\left(N_{, 1}\right)^{2}\left\langle\phi_{1} \phi_{1}\right\rangle+2\left(N_{, 1}\right)\left(N_{, 2}\right)\left\langle\phi_{1} \phi_{2}\right\rangle \\
& +\left(N_{, 2}\right)^{2}\left\langle\phi_{2} \phi_{2}\right\rangle,
\end{aligned}
$$

where we have allowed for cross correlation between the fields - for a canonical kinetic term, the cross coupling is zero. We see, then, that the power spectrum receives extra 
contributions in multifield inflation, both from cross couplings and from the extra two point correlation functions of the additional fields.

We would like to analyze these extra contributions in more detail. Using the approximation that the DBI multifield parameters (10) are small and that the potential is separable $V\left(\phi_{1}, \phi_{2}\right)=V_{1}\left(\phi_{1}\right)+V_{2}\left(\phi_{2}\right)$ and dominates the energy density, the number of e-folds from the time of horizon crossing to the end of inflation is [10]

$$
N_{e}=-\frac{1}{M_{p}^{2}} \int_{*}^{e} \frac{V_{1}}{c_{s} \partial_{\phi_{1}} V_{1}} d \phi_{1}-\frac{1}{M_{p}^{2}} \int_{*}^{e} \frac{V_{2}}{c_{s} \partial_{\phi_{2}} V_{2}} d \phi_{2} \text {. }
$$

As in [10], we can write the curvature perturbation (19) in a much simpler way by using a different set of multifield DBI parameters,

$$
\epsilon_{i}=\frac{c_{s} M_{p}^{2}}{2}\left(\frac{V_{i}^{\prime}}{V}\right)^{2}
$$

where a prime denotes a derivative with respect to the argument. Note that $\epsilon=-\dot{H} / H^{2}=\epsilon_{1}+\epsilon_{2}$. The curvature perturbation can now be written,

$$
\begin{aligned}
\zeta= & \frac{1}{M_{p} \sqrt{2 \epsilon_{1}^{*}}}\left(\frac{V_{1}^{*}+Z^{e}}{V^{*}}\right)\left(\delta \phi_{1}\right)^{*} \\
& +\frac{1}{M_{p} \sqrt{2 \epsilon_{2}^{*}}}\left(\frac{V_{2}^{*}-Z^{e}}{V^{*}}\right)\left(\delta \phi_{2}\right)^{*},
\end{aligned}
$$

with

$$
Z^{e} \equiv \frac{V_{2}^{e} \epsilon_{1}^{e}-V_{1}^{e} \epsilon_{2}^{e}}{\epsilon^{e}}
$$

where the superscript " $e$ " denotes evaluation at the end of inflation. In the limit that the trajectory is in the radial or angular direction as shown above then at the end of inflation the one of the field has settled into its minima (say, $\phi_{2}$ for concreteness) so $Z^{e}=V_{2}^{e}=$ const. For massterm dominated potentials (or equivalently when the vacuum energy from $\phi_{2}$ is small $V_{2}^{e} \ll V_{2}^{*}$ ) then we can take $Z^{e}=0$ and the expression for the curvature perturbation simplifies to include only the values of the potential and the slow-roll parameters evaluated at horizon crossing,

$$
\zeta=\frac{1}{M_{p} \sqrt{2 \epsilon_{1}^{*}}}\left(\frac{V_{1}^{*}}{V^{*}}\right)\left(\delta \phi_{1}\right)^{*}+\frac{1}{M_{p} \sqrt{2 \epsilon_{2}^{*}}}\left(\frac{V_{2}^{*}}{V^{*}}\right)\left(\delta \phi_{2}\right)^{*} .
$$

This simple expression for the curvature perturbation will be useful later in evaluating the power spectrum.

\section{A. The inflationary perturbation}

In order to calculate the power spectrum we need to evaluate the two point correlation functions for the perturbations of the scalar fields in (20). In order to have analytic control over our expressions we will assume that the trajectory is highly radial, e.g.

$$
\tan \theta=\frac{\dot{\phi}_{2}}{\dot{\phi}_{1}}=\frac{V_{2}^{\prime}}{V_{1}^{\prime}} \ll c_{s} .
$$

Under this assumption, the adiabatic perturbation is simply the perturbation in the $\phi_{1}$ direction and perturbations in the $\phi_{2}$ direction are just isocurvature perturbations,

$$
\delta \sigma=\delta \phi_{1}, \quad \delta s=\delta \phi_{2} .
$$

Expanding the kinetic part of the Lagrangian in (2) to quadratic order in $\delta \sigma$ and $\delta s$, we find

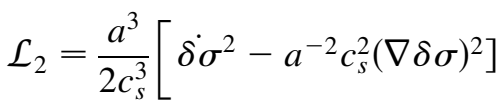

$$
\begin{aligned}
& +\frac{a^{3}}{2 c_{s}}\left[\dot{\delta} s^{2}-a^{-2}(\nabla \delta s)^{2}\right]
\end{aligned}
$$

Note that the isocurvature fluctuations (e.g. the fluctuations in the angular direction) scale differently with the sound speed; we will see soon that this has important consequences for the two point functions.

The quantization of the perturbations proceeds as usual with

$$
\begin{aligned}
\delta \sigma(\tau, \mathbf{x})= & \frac{1}{(2 \pi)^{3}} \int d^{3} k[u(\tau, \mathbf{k}) a(\mathbf{k}) \\
& \left.+u^{*}(\tau,-\mathbf{k}) a^{\dagger}(-\mathbf{k})\right] e^{i \mathbf{k} \cdot \mathbf{x}}, \\
\text { where } u(\tau, \mathbf{k})= & \frac{H}{\sqrt{2 k^{3}}}\left(1+i k c_{s} \tau\right) e^{-i k c_{s} \tau}
\end{aligned}
$$

for the adiabatic perturbation and

$$
\begin{aligned}
\delta s(\tau, \mathbf{x})= & \frac{1}{(2 \pi)^{3}} \int d^{3} k[v(\tau, \mathbf{k}) b(\mathbf{k}) \\
& \left.+v^{*}(\tau,-\mathbf{k}) b^{\dagger}(-\mathbf{k})\right] e^{i \mathbf{k} \cdot \mathbf{x}},
\end{aligned}
$$

where $v(\tau, \mathbf{k})=H \sqrt{\frac{c_{s}^{-}}{2 k^{3}}}(1+i k \tau) e^{-i k \tau}$

for the isocurvature modes. The creation and annihilation operators satisfy the usual commutation relation $\left[a(\mathbf{k}), a^{\dagger}\left(\mathbf{k}^{\prime}\right)\right]=\left[b(\mathbf{k}), b^{\dagger}\left(\mathbf{k}^{\prime}\right)\right]=(2 \pi)^{3} \delta^{3}\left(\mathbf{k}-\mathbf{k}^{\prime}\right), u(\tau, \mathbf{k})$ and $v(\tau, \mathbf{k})$ are the solutions of the quadratic Lagrangian whose normalizations are fixed by the Wronskian conditions, ${ }^{1}$ and $\tau=-\frac{1}{a H}$ is the conformal time.

It is now straightforward to calculate the two point functions,

$$
\begin{aligned}
& \left\langle\delta \sigma\left(\mathbf{k}_{1}\right) \delta \sigma\left(\mathbf{k}_{2}\right)\right\rangle=(2 \pi)^{3} \delta^{3}\left(\mathbf{k}_{1}+\mathbf{k}_{2}\right) \frac{H^{2}}{2 k_{1}^{3}}, \\
& \left\langle\delta s\left(\mathbf{k}_{1}\right) \delta s\left(\mathbf{k}_{2}\right)\right\rangle=(2 \pi)^{3} \delta^{3}\left(\mathbf{k}_{1}+\mathbf{k}_{2}\right) \frac{c_{s} H^{2}}{2 k_{1}^{3}} .
\end{aligned}
$$

\footnotetext{
${ }^{1}$ This can be checked by computing the commutator $\left[\delta \sigma\left(\tau, \mathbf{x}_{1}\right), p_{\delta \sigma}\left(\tau, \mathbf{x}_{2}\right)\right]=i \delta^{3}\left(\mathbf{x}_{1}-\mathbf{x}_{2}\right)$, where $p_{\delta \sigma}=\frac{\partial \mathcal{L}_{2}}{\partial \delta \sigma}$ is the canonical momentum.
} 
Here $\delta \sigma(\mathbf{k})=\left.\delta \sigma(\tau, \mathbf{k})\right|_{\tau \rightarrow 0}, \delta s(\mathbf{k})=\left.\delta s(\tau, \mathbf{k})\right|_{\tau \rightarrow 0}$, and the Hubble parameter and sound speed are evaluated at the time of horizon crossing. Here we see that the isocurvature fluctuations generated by $\delta s$ are suppressed by a factor of $c_{s} \ll 1$ compared to the adiabatic perturbations generated by $\delta \sigma$. This suppression can be traced back to the different $c_{s}$ dependence found in the quadratic Lagrangian (28). We note that the exact same calculation follows through if the trajectory is dominated by the angular direction after the replacement $\delta \phi_{1} \leftrightarrow \delta \phi_{2}$. Finally, notice also that in the limit of a straightline trajectory there is no cross correlation between the fields, so the cross term in the power spectrum (20) vanishes. Unfortunately, nonstraight line trajectories are beyond our analytic control so it is not clear if the cross coupling will be significant, although we expect that in the diagonal limit the two point function should reduce to that of a simple single field model where the results are well known.

Using the two point functions (31) and (32) and the expression for the curvature perturbation (25), the power spectrum (20) becomes,

$$
\begin{aligned}
P_{\zeta} & =\frac{H^{2}}{4 \pi^{2} M_{p}^{2}}\left[\frac{1}{2 \epsilon_{1}^{*}}\left(\frac{V_{1}^{*}}{V^{*}}\right)^{2}+\frac{c_{s}}{2 \epsilon_{2}^{*}}\left(\frac{V_{2}^{*}}{V^{*}}\right)^{2}\right] \\
& \approx \frac{H^{2}}{4 \pi^{2} M_{p}^{2}} \frac{1}{2 \epsilon_{1}^{*}}\left(\frac{V_{1}^{*}}{V^{*}}\right)^{2}
\end{aligned}
$$

where in the last line we assume that the second term is small compared to the first term in the small $c_{s}$ limit.

Since the contribution of the angular modes to the power spectrum is highly suppressed by the sound speed we find that multifield DBI reduces essentially to the single field case, in contrast to multiple field slow-roll inflation where additional fields may become important when the trajectory makes sharp turns in field space [10].

\section{B. Multifield non-Gaussianity}

In the following we study the non-Gaussianities in more details, and as it turns out there are some potentially observable differences from the single field DBI inflation. To compute the non-Gaussianities, we expand the DBI Lagrangian to higher order. The leading order and subleading order cubic terms are

$$
\begin{aligned}
\mathcal{L}_{3}= & \frac{a^{3}}{2 c_{s}^{5} \dot{\sigma}}\left[\dot{\delta} \dot{\sigma}^{3}-a^{-2} c_{s}^{2} \dot{\delta} \sigma(\nabla \delta \sigma)^{2}\right. \\
& \left.-a^{-2} c_{s}^{2} \dot{\delta} \sigma(\nabla \delta s)^{2}\right]
\end{aligned}
$$

The leading contribution to the non-Gaussianity comes from the first two terms, and their size is well known

$$
f_{\mathrm{NL}} \sim \frac{1}{c_{s}^{2}}
$$

Since the angular mode $\delta s$ is suppressed by a factor of $\sqrt{c_{s}}$ comparing to the radial mode $\delta \sigma$, we see that the third term in (34) contributes a non-Gaussianity of order

$$
f_{\mathrm{NL}} \sim \frac{1}{c_{s}}
$$

Although it is subleading comparing to the first two terms, it is still potentially observable by future experiments if the sound speed $c_{s}$ is small enough. In particular, this effect can be larger than the subleading effect of order $\frac{\epsilon}{c_{s}^{2}}$ computed in [4] when the sound speed satisfies $c_{s}>\epsilon$.

The calculation of the three-point function is standard, see e.g. [4] for details,

$$
\begin{aligned}
\left\langle\zeta\left(\mathbf{k}_{1}\right) \zeta\left(\mathbf{k}_{2}\right) \zeta\left(\mathbf{k}_{3}\right)\right\rangle= & -i(2 \pi)^{3} \delta^{3}\left(\sum_{i} \mathbf{k}_{i}\right) v\left(0, \mathbf{k}_{1}\right) v\left(0, \mathbf{k}_{2}\right) u\left(0, \mathbf{k}_{3}\right)\left(\frac{\partial N}{\partial \phi_{1}^{*}}\right)\left(\frac{\partial N}{\partial \phi_{2}^{*}}\right)^{2} \frac{1}{c_{s}^{3} \dot{\sigma}} \\
& \times \int_{-\infty}^{0} a d \tau \frac{d u^{*}\left(\tau, \mathbf{k}_{3}\right)}{d \tau}\left[\left(-\mathbf{k}_{1} \cdot \mathbf{k}_{2}\right) v^{*}\left(\tau, \mathbf{k}_{1}\right) v^{*}\left(\tau, \mathbf{k}_{2}\right)\right]+\text { c.c. }+ \text { cyclic. }
\end{aligned}
$$

here "cyclic." means two other terms by cyclically permuting $k_{1}, k_{2}, k_{3}$. We can see the non-Gaussianity vanishes in the squeezed limit when one of the momentum $k_{i} \rightarrow 0$, since $\frac{d u^{*}\left(\tau, \mathbf{k}_{3}\right)}{d \tau} \sim k_{3}^{2}$ and there is factor of $\mathbf{k}_{1} \cdot \mathbf{k}_{2}$. This is the same as in single field DBI inflation.

Going away from the squeezed limit, we can compute the above three-point function assuming $k_{1}, k_{2}, k_{3}$ are of the same order of magnitude. The non-Gaussianity has a very interesting shape as the following

$$
\begin{aligned}
\mathcal{A}\left(k_{1}, k_{2}, k_{3}\right)= & \frac{k_{3}^{2}\left(k_{1}^{2}+3 k_{1} k_{2}+k_{2}^{2}\right)\left(-\mathbf{k}_{1} \cdot \mathbf{k}_{2}\right)}{\left(k_{1}+k_{2}\right)^{3}} \\
& + \text { cyclic. }
\end{aligned}
$$

We plot the non-Gaussianity as $\mathcal{A}\left(k_{1}, k_{2}, 1\right) /\left(k_{1} k_{2}\right)$ following the convention of [4] in Fig. 3.

We can see from Fig. 4 that the non-Gaussianity has a very interesting new feature, namely, the sign of nonGaussianity is different in the middle of the "folded triangle" limit where $k_{3}=k_{1}+k_{2}$ from most of the other region of the configuration space. For example, one can directly check for the configuration of a folded triangle $k_{1}=k_{2}=\frac{k_{3}}{2}$, the shape is negative $\mathcal{A}\left(k_{1}, k_{2}, k_{3}\right)=$ $-\frac{1.088}{k_{3}^{3}}$. This feature is not present in any other known inflationary models, so it can be used as a distinctive signature of multifield DBI inflation. The sign of nonGaussianity determines the sense of skewness in the 


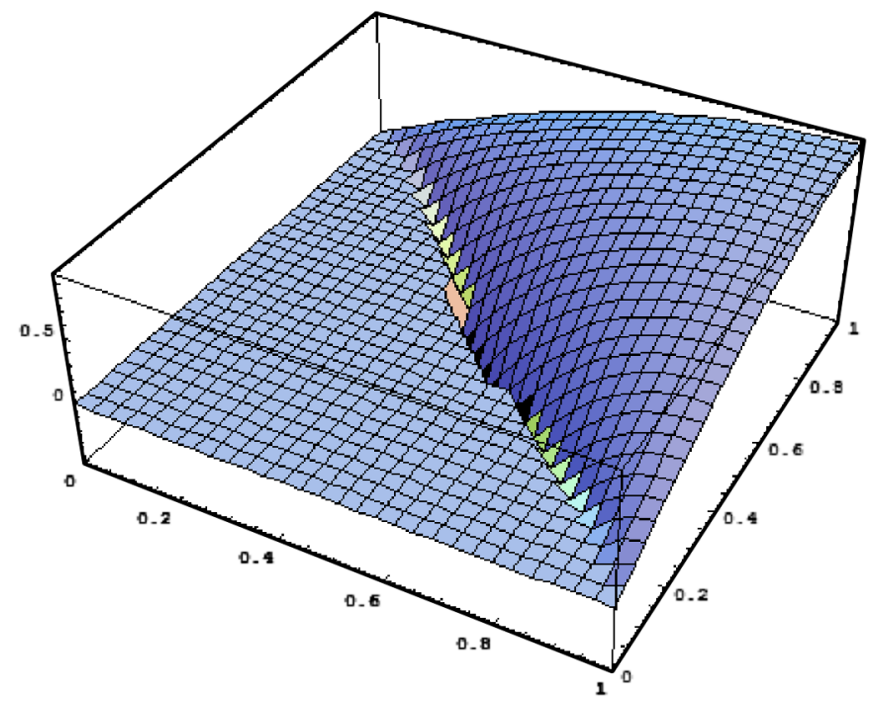

FIG. 3 (color online). The shape of non-Gaussianity in multifield DBI inflation is shown through a plot of $\mathcal{A}\left(k_{1}, k_{2}, 1\right) /\left(k_{1} k_{2}\right)$ in Eq. (38). Notice that in the folded triangle limit $k_{1}=k_{2}=$ $k_{3} / 2$ the bispectrum is negative, and constitutes a distinctive signature of multifield DBI inflation in the small $c_{s}$ limit. The presence of opposite signs of the non-Gaussianity may give rise to interesting observational effects.

CMB temperature and matter density, and thus this change in sign can lead to potentially interesting observational effects. ${ }^{2}$ However, we should caution that since this is a subleading effect, it might be difficult to disentangle the signature from experimental data. It would be interesting to extend this analysis to more than two fields, which may lead to additional enhancements or suppressions of the multifield non-Gaussianities [12].

\section{Discussion}

We have shown that the two point function of the extra "angular" scalar field direction during inflation is suppressed by a factor of $c_{s}$ compared to the usual single field contribution, thus the multifield DBI observables simply reduce to the single field case (in the limit where one of the fields dominates the trajectory). There have been a lot of recent attempts in building single field DBI inflation models consistent with all known compactification constraints and precision cosmological observations [13]. In particular, these works find that combining observational constraints of the amplitudes and tilt of the scalar and tensor

\footnotetext{
${ }^{2}$ The convention for the sign of $f_{N L}$ (which characterizes the level of non-Gaussianity) has been a source of confusion in the literature. See [11] for a discussion.
}

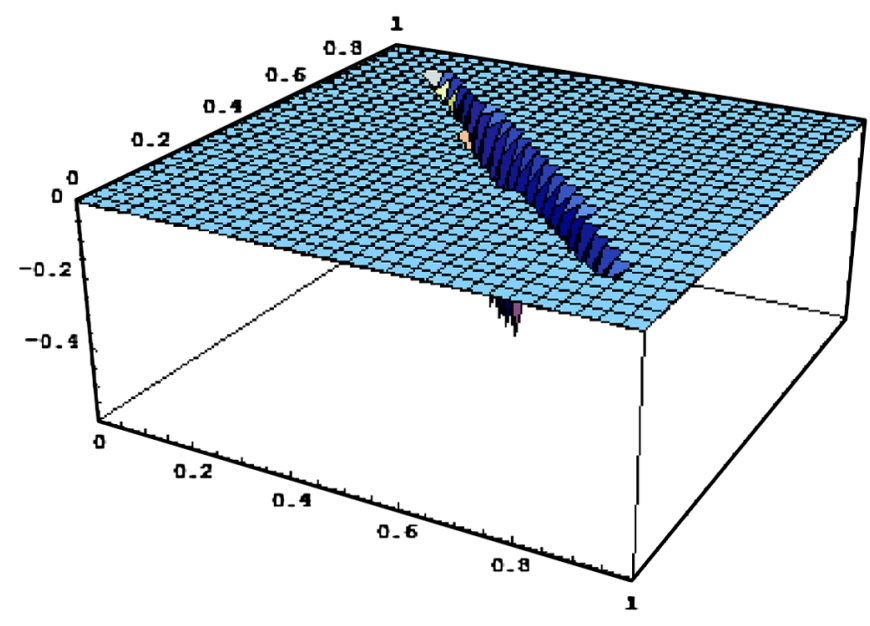

FIG. 4 (color online). The negative part of the non-Gaussianity in the folded triangle limit is shown.

perturbations and the primordial non-Gaussianity together with limitations on the field range coming from compactification puts severe constraints on the viable parameter space of single field DBI models, ruling out the most simple models.

In general, since we see that the small sound speed in multifield DBI inflation suppresses the multifield effects, multifield DBI inflationary observables can be well approximated by their single field values. Note that this also implies that the dramatic $\mathcal{O}(1)$ effects expected at the end of multifield DBI inflation due to the inhomogenous surface of tachyon condensation examined in [14] are now instead suppressed by $\mathcal{O}\left(c_{s}\right)$ and are subdominant.

Variations of the basic DBI model, such as its IR version $[15,16]$, models involving wrapped branes [17] or different warped geometries, may be able to evade the strong constraints of [13]; since we have not made explicit use of any particular model we expect our results to hold in the small sound speed limit of these models as well.

\section{ACKNOWLEDGMENTS}

We would like to thank Thorsten Battefeld, Damien Easson, Louis Leblond, Liam McAllister, Sarah Shandera and Gianmassimo Tasinato for helpful discussions and comments. The work of M.H., G.S. and B. U. was supported in part by NSF CAREER Grant No. PHY-0348093, DOE grant DE-FG-02-95ER40896, and by Research Corporation.

Note added-As this manuscript was being prepared, a preprint [18] appeared which contains some overlap with this work. 
[1] J. Garriga and V.F. Mukhanov, Phys. Lett. B 458, 219 (1999); C. Armendariz-Picon, T. Damour, and V.F. Mukhanov, Phys. Lett. B 458, 209 (1999).

[2] E. Silverstein and D. Tong, Phys. Rev. D 70, 103505 (2004).

[3] M. Alishahiha, E. Silverstein, and D. Tong, Phys. Rev. D 70, 123505 (2004).

[4] X. Chen, M. x. Huang, S. Kachru, and G. Shiu, J. Cosmol. Astropart. Phys. 01 (2007) 002; M.x. Huang and G. Shiu, Phys. Rev. D 74, 121301 (2006).

[5] G. R. Dvali and S. H. H. Tye, Phys. Lett. B 450, 72 (1999).

[6] S. B. Giddings, S. Kachru, and J. Polchinski, Phys. Rev. D 66, 106006 (2002).

[7] I. R. Klebanov and M. J. Strassler, J. High Energy Phys. 08 (2000) 052.

[8] C. Gordon, D. Wands, B. A. Bassett, and R. Maartens, Phys. Rev. D 63, 023506 (2000).

[9] M. Sasaki and E. D. Stewart, Prog. Theor. Phys. 95, 71 (1996); A. A. Starobinsky, Pis'ma Zh. Eksp. Teor. Fiz. 42, 124 (1985) [JETP Lett. 42, 152 (1985)]; D. Wands, K. A. Malik, D. H. Lyth, and A. R. Liddle, Phys. Rev. D 62, 043527 (2000); D. Seery and J.E. Lidsey, J. Cosmol. Astropart. Phys. 09 (2005) 011.

[10] F. Vernizzi and D. Wands, J. Cosmol. Astropart. Phys. 05 (2006) 019.
[11] E. Komatsu, Cosmology and Strings Workshop, ICTP, Trieste, 2007.

[12] T. Battefeld and R. Easther, J. Cosmol. Astropart. Phys. 03 (2007) 020; D. Battefeld and T. Battefeld, J. Cosmol. Astropart. Phys. 05 (2007) 012.

[13] S. Kecskemeti, J. Maiden, G. Shiu, and B. Underwood, J. High Energy Phys. 09 (2006) 076; G. Shiu and B. Underwood, Phys. Rev. Lett. 98, 051301 (2007); D. Baumann and L. McAllister, Phys. Rev. D 75, 123508 (2007); J. E. Lidsey and I. Huston, J. Cosmol. Astropart. Phys. 07 (2007) 002; R. Bean, S. E. Shandera, S. H. H. Tye, and J. Xu, J. Cosmol. Astropart. Phys. 05 (2007) 004; H. V. Peiris, D. Baumann, B. Friedman, and A. Cooray, Phys. Rev. D 76, 103517 (2007);

[14] L. Leblond and S. Shandera, J. Cosmol. Astropart. Phys. 01 (2007) 009.

[15] X. Chen, J. High Energy Phys. 08 (2005) 045; X. Chen, Phys. Rev. D 72, 123518 (2005).

[16] S. Thomas and J. Ward, Phys. Rev. D 76, 023509 (2007).

[17] T. Kobayashi, S. Mukohyama, and S. Kinoshita, arXiv:0708.4285; M. Becker, L. Leblond, and S.E. Shandera, Phys. Rev. D 76, 123516 (2007).

[18] D. A. Easson, R. Gregory, D. F. Mota, G. Tasinato, and I. Zavala, arXiv:0709.2666. 